\title{
A Comparative Study of Greenfield Tunnelling in Sands: FEM, DEM, and Centrifuge Modelling
}

Song, Geyang; Franza, Andrea; Elkayam, Itai; Marshall, Alec; Klar, Assaf

Published in:

Micro to MACRO Mathematical Modelling in Soil Mechanics

Link to article, DOI:

10.1007/978-3-319-99474-1_34

Publication date:

2019

Document Version

Peer reviewed version

Link back to DTU Orbit

Citation (APA):

Song, G., Franza, A., Elkayam, I., Marshall, A., \& Klar, A. (2019). A Comparative Study of Greenfield Tunnelling in Sands: FEM, DEM, and Centrifuge Modelling. In Micro to MACRO Mathematical Modelling in Soil Mechanics (pp. 337-345). Springer. Trends in Mathematics https://doi.org/10.1007/978-3-319-99474-134

\section{General rights}

Copyright and moral rights for the publications made accessible in the public portal are retained by the authors and/or other copyright owners and it is a condition of accessing publications that users recognise and abide by the legal requirements associated with these rights.

- Users may download and print one copy of any publication from the public portal for the purpose of private study or research.

- You may not further distribute the material or use it for any profit-making activity or commercial gain

- You may freely distribute the URL identifying the publication in the public portal 


\title{
A study of greenfield tunnelling in sands using FEM, DEM, and centrifuge modelling
}

\author{
Geyang Song, Andrea Franza, Itai Elkayam, Alec M. \\ Marshall and Assaf Klar
}

\begin{abstract}
The effect of tunnel construction on ground displacements is an important problem for tunnelling engineers. Numerical methods, including continuum and discrete element methods, have been used to evaluate tunnelling induced ground displacements. In this paper, the ability of numerical methods to replicate the response to tunnelling of a real soil is evaluated by comparing results from numerical analyses with experimental data obtained from geotechnical centrifuge tests. The centrifuge tests include two types of tunnel boundary condition: pressure-controlled (water extracted from model tunnel within a flexible membrane) or displacement controlled (rigid boundary model tunnel undergoing an eccentric contraction). Centrifuge measurements are compared against discrete element method (DEM) and finite element method (FEM) analyses which replicate the conditions of the experiments: pressure controlled boundary for FEM and DEM; displacement controlled boundary for FEM only. The effects of tunnel boundary condition on the soil displacement mechanisms are illustrated and the performance of the numerical analyses to replicate salient features of ground response are discussed.
\end{abstract}

Keywords. discrete element method (DEM), finite element method (FEM), tunnelling, centrifuge.

\section{INTRODUCTION}

The prediction of ground movements caused by tunnelling is important in order to evaluate potential damage to structures and critical infrastructure. Tunnelling induced displacements are often modelled as a simplified plane strain problem, where tunnel advancement is not modelled. Induced ground displacements at the tunnel are replicated through a tunnel volume loss, $V_{l, t}$, which indicates the ratio of ground lost at the tunnel periphery to the notional cross-sectional area of 
the tunnel, expressed as a percentage. Tunnelling induced soil movements have been investigated experimentally by a number of researchers using geotechnical centrifuge testing, where a small-scale model is put within an environment of increased acceleration ('gravity'), thereby increasing the self-weight of the model soil and replicating the behaviour of a full-scale prototype. The most popular method for replicating tunnel volume loss in a centrifuge environment is with a fluid filled cylindrical model tunnel surrounded by a flexible membrane (FM), whereby extraction of a volume of water from the model tunnel initiates ground movements around the tunnel. An alternative approach is to use a rigid boundary mechanical (RBM) model tunnel, as developed by Boonsiri and Takemura [1], where displacements were imposed concentrically around the tunnel circumference. Recently, Song et al. [2] developed an eccentric RBM model tunnel (referred to here as eRBM), where an eccentric profile of displacements is created (max displacements at tunnel crown, no displacements at invert) which matches better to real ground displacements around shallow tunnels. Numerical modelling has been used extensively to simulate tunnelling problems. Finite element method (FEM) analyses are probably the most popular numerical tool for this purpose. As with centrifuge testing, there are different ways in which tunnel volume loss can be simulated. In this paper, results are obtained using the pressure controlled method (PCM) [3] and the displacement controlled method (DCM) [4] and compared against centrifuge test data. The PCM gradually reduces the internal pressure within the FEM model tunnel and can be used to simulate FM centrifuge tests. The DCM imposes predefined displacements at the boundary of the FEM model tunnel; it can be used to simulate RBM centrifuge tests.

Numerical modelling of tunnels using the discrete element method (DEM) has not seen as much attention as the FEM due to the computational demands of the method, however there are some cases where DEM has been used (for example, Marshall et al. [5] compared DEM results of greenfield tunnelling against centrifuge test data).

In this paper, greenfield tunnelling soil displacements from plane-strain geotechnical centrifuge tests are compared against FEM (using ABAQUS) and DEM (using $\mathrm{PFC}^{3 \mathrm{D}}$ ) numerical simulations designed to replicate the centrifuge tests. During the centrifuge tests, both flexible membrane (FM) and eccentric rigid boundary mechanical (eRBM) model tunnels were used. The PCM was applied within both the FEM and DEM models (replicating flexible membrane centrifuge model tunnels); the DCM was applied only within the FEM models (replicating the eRBM model tunnel).

\section{EXPERIMENTAL AND NUMERICAL MODELS}

\subsection{Centrifuge models}

The centrifuge tests were performed on the University of Nottingham Centre for Geomechanics (NCG) 4m diameter 50 g-ton geotechnical centrifuge. Tests were 
conducted at an acceleration of 80 times gravity. The eRBM model tunnel [2] consists of six independent segments extend along the tunnel axis length that could move inwards at different rates during tunnel volume loss, thereby creating a non-uniform profile of displacements around the tunnel circumference (max displacements at crown, zero at tunnel invert). The FM model tunnel consists of a water-filled cylindrical flexible membrane sealed at each end of the tunnel [6]. Volume loss is simulated by gradually extracting water from the tunnel. In both the eRBM and FM model tunnels, a plane-strain scenario is modelled where displacements are uniform along the tunnel length.

The centrifuge models include an acrylic front window to allow acquisition of images of the sub-surface such that image analysis techniques can be used to evaluate soil movements. Comparison of displacements from image analysis at the model boundary against measurements made at the middle of the model width (using traditional displacement transducers) have shown that boundary friction has a minimal effect on settlement trough shape [6].

The soil used in all tests was dry Leighton Buzzard Fraction E silica sand, which has a typical $D_{50}$ of $0.12 \mathrm{~mm}$, a specific gravity of 2.65 , and maximum:minimum void ratios of 1.01:0.61 [7]. The sand model was prepared by dry air pluviation to achieve a relative density $\left(I_{d}\right)$ of approximately $90 \%$ (both FM and eRBM tests).

\subsection{FEM models}

Two-dimensional finite element (FE) analyses were performed using ABAQUS. Due to symmetry, only half of the model was considered. Eight-node quadratic plane-strain elements (CPE8) were used for the tests. Vertical roller boundaries were used on the plane of symmetry and side boundaries, a fixed boundary was used for the bottom, and no constraints were imposed at the soil surface. The dimensions of the numerical model were chosen to minimise boundary effects on ground displacements, and the element size was verified to have a negligible effect on results. The basic hypoplastic model developed by Gudehus and Masin [8] was used. The eight parameters of the constitutive model were calibrated by simulating oedometer (stress-void ratio curve fitting) and triaxial (stress-strain curve fitting) test data provided by Lanzano et al. [9] for Leighton Buzzard Fraction E sand. The calibrated parameters are: critical state friction angle $\phi_{c}=33.4^{\circ}$, granulate hardness $h_{s}=2402 \mathrm{MPa}$, exponent $n=0.365$, minimum:maximum void ratio at zero pressure $e_{d 0}: e_{i 0}=0.55: 1.21$, critical void ratio at zero pressure $e_{c 0}=1.01$, and exponents $\alpha=0.1, \beta=1.0$.

In the FE analyses, the displacement-controlled method (DCM) [4] was used to simulate the eRBM centrifuge model tunnel. FE nodal displacements were imposed that coincided with the centrifuge eRBM model tunnel displacements. FE analyses using the pressure-controlled method (PCM) were also conducted, whereby the internal pressure within the FE model tunnel was gradually reduced until a specified magnitude of tunnel volume loss was achieved. This process simulated the extraction of water (and resultant reduction of pressure) from within the FM centrifuge model tunnel. It is assumed that in the centrifuge tests the initial 
at-rest earth pressure coefficient $K_{0}$ is 0.5 . This assumption was also adopted for the numerical analyses, however data from an additional analysis where $K_{0}=0.8$ is also presented.

\subsection{DEM models}

DEM simulations, performed using $\mathrm{PFC}^{3 \mathrm{D}}$ by Itasca, simulated as close as possible the conditions within the FM centrifuge experiments (see Marshall et al. [5] for full details). Note that DEM was not used to simulate the eRBM experiments. The uniform particle size had a radius of $2.5 \mathrm{~mm}$ to simulate the Fraction E sand used in the centrifuge test. The Hertz-Mindlin contact and slip models were implemented into the simulation. The spherical particles (balls) had a density $\rho=2600 \mathrm{~kg} / \mathrm{m}^{3}$, shear stiffness $G=31 \times 10^{7} \mathrm{kPa}$, Poisson's ratio $v=0.27$ and inter-particle friction coefficient $\mu=0.5$. The wall boundaries were assumed frictionless.

The specified relative density was achieved by dropping the balls under $1 \mathrm{~g}$ to fill the box, followed by reducing the friction coefficient while the particles to be compacted (using a horizontal wall) until the required relative density was obtained. Once equilibrium was achieved, the original coefficient of friction was re-imposed and the gravity was increased to the level within the centrifuge tests.

The FM centrifuge model tunnel was represented in the DEM model by a series of 50 (around the tunnel circumference) smooth rectangular wall elements which extended along the length of the tunnel. The segments could only move radially towards the centre of the tunnel axis. The displacement of the wall elements was controlled by a subroutine which replicated the removal of water from the centrifuge model tunnel [5].

\section{RESULTS}

Figure 1 shows vertical and horizontal displacements for all of the models at a tunnel volume loss of $2 \%$. Note that displacements are presented in model scale; full-scale values can be obtained by scaling by $N=80$. Comparing the FM and eRBM centrifuge model displacements in Figure 1a-b and f-g, vertical displacements are more localised in the region above the tunnel crown for the FM test than the eRBM; in the eRBM test the contours of vertical displacements initiate from the area around the side of the tunnel. The pattern of horizontal displacements in the FM and eRBM centrifuge test data is noticeably different, with a localised zone at the side of the tunnel in the eRBM test that is not apparent in the FM test. In general, the centrifuge data indicate that in the FM test, displacements are initiated mostly at the tunnel crown, whereas in the eRBM test, displacements are distributed more evenly around the upper half of the tunnel, with much more horizontal displacement occurring at the side of the tunnel than in the FM test.

Considering the FEM results in Figure 1c-d and h-i, the DCM analyses show a much more localised zone of vertical displacement around the tunnel crown then the PCM results, and the magnitude of vertical displacement within most 

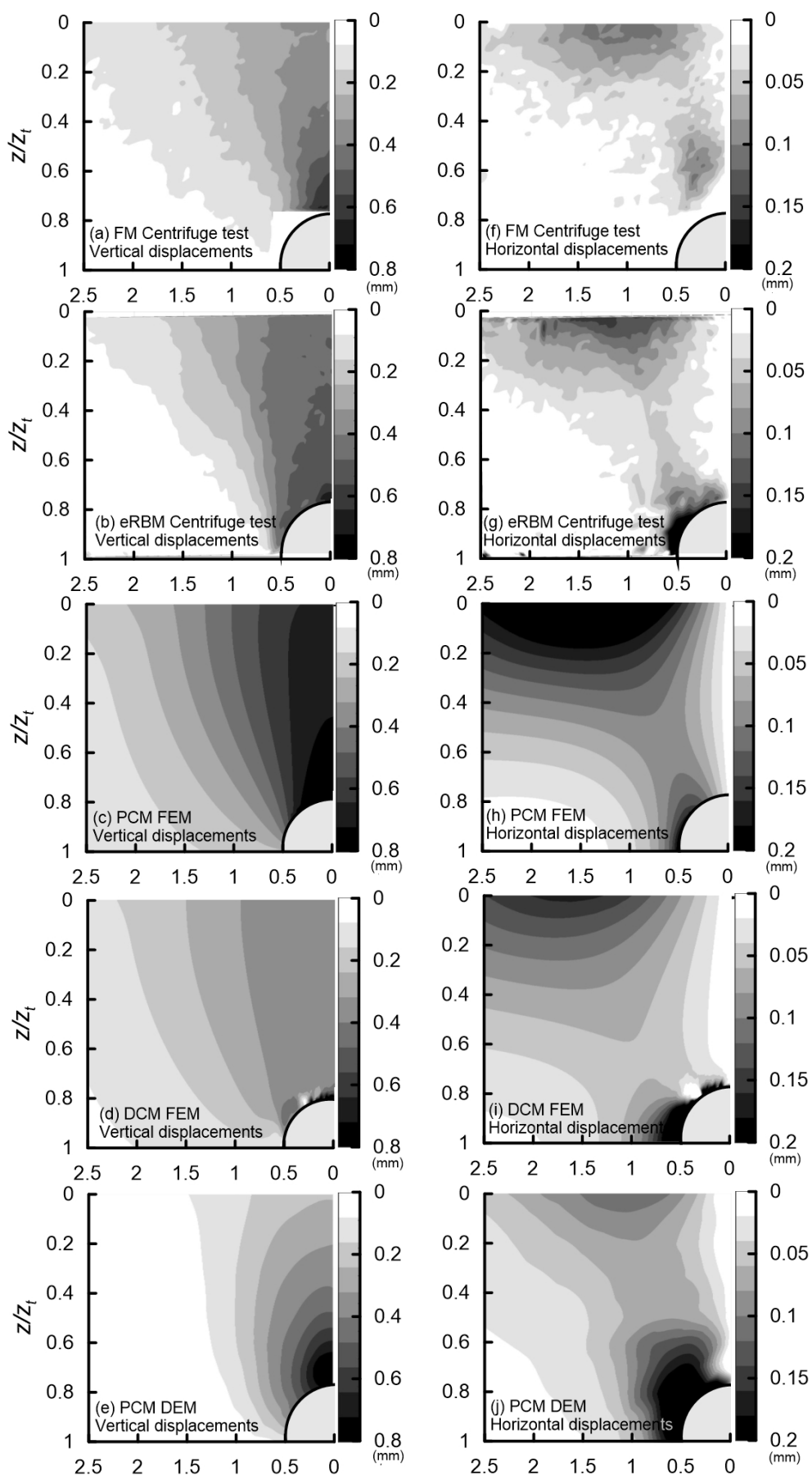

FiguRE 1. Vertical (a-e) and horizontal (f-j) soil displacements from all models. $C / D=2 ; V_{l, t}=2 \% ; I_{d}=90 \%$; displacements downwards and to the right are positive) 
of the soil is much less for the DCM. In terms of horizontal displacements, the DCM and PCM patterns are similar, however the localised zone of horizontal displacements at the side of the DCM tunnel is larger than for the PCM tunnel, whereas the magnitudes of horizontal displacement near the surface are greater for the PCM tunnel than the DCM. Comparison of both the PCM and DCM FEM results against the centrifuge data indicates that the FEM models predict more significant displacements in a much larger zone of soil around the tunnel than observed experimentally.

The PCM DEM analyses (e and $\mathrm{j}$ ), which simulate the FM centrifuge test, provide a good overall match to the distribution of vertical displacements from the centrifuge tests; the affected zone is much narrower than in the FEM analyses. It's not possible to conclude that the PCM DEM model matches better to the FM centrifuge test data (which it was designed to replicate) than the eRBM data; the PCM DEM results include features present in both the FM and eRBM centrifuge tests (e.g. localised horizontal displacements at the side of the tunnel, as in the eRBM). It would be interesting to compare results from an equivalent DCM DEM analysis which replicated the eRBM centrifuge test; this will form the basis of future work in this area.

Of interest to tunnel engineers is the magnitude and shape of the settlement trough caused by tunnelling. Figure 2 plots the settlement trough data $\left(S_{v}\right)$ and the normalised settlements $\left(S_{v} / S_{\max }\right.$, where $S_{\max }$ is maximum settlement) from all the models at the ground surface at at a normalised depth of $z / z_{t}=0.5$. Considering first the centrifuge test data, the magnitude of $S_{\max }$ is shown to be similar for the eRBM and FM tests (Figure 2a-b), but there is a difference in settlement trough shape (most notable at the subsurface in Figure 2d). The eRBM model tunnel creates a flatter profile in the area above the tunnel, with an adjacent zone where settlements decrease rapidly.

The PCM FEM analysis with $K_{0}=0.5$ (replicating the assumed initial state of the centrifuge tests) over-predicts the surface and subsurface settlements (Figure 2a-b). By adopting a $K_{0}$ of 0.8 , the magnitude of settlements is reduced closer to the centrifuge test data. Focusing on the predicted FEM settlement trough shapes in Figure 2c-d, there is very little difference between the displacement and pressure controlled analyses (both $K_{0}=0.5$ and 0.8 ). The FEM predictions match reasonably well to the experimental data at the surface, however at greater depths, the FEM predictions are much 'shallower' than the experimental data.

The results in Figure 2a-b indicate that the DEM model over-predicts the amount of dilation experienced by the soil. Whilst the settlements at $z / z_{t}=0.5$ match the experimental data rather well, at the surface the DEM settlements are notably lower than the experimental data, indicating that the soil is dilating and decreasing the magnitude of soil volume loss towards the surface. Figure 2c-d shows that the DEM model does a better job at predicting the 'sharp' settlement trough from the experiments than the FEM analyses. The DEM profile matches better to the FM centrifuge data than the eRBM data. 

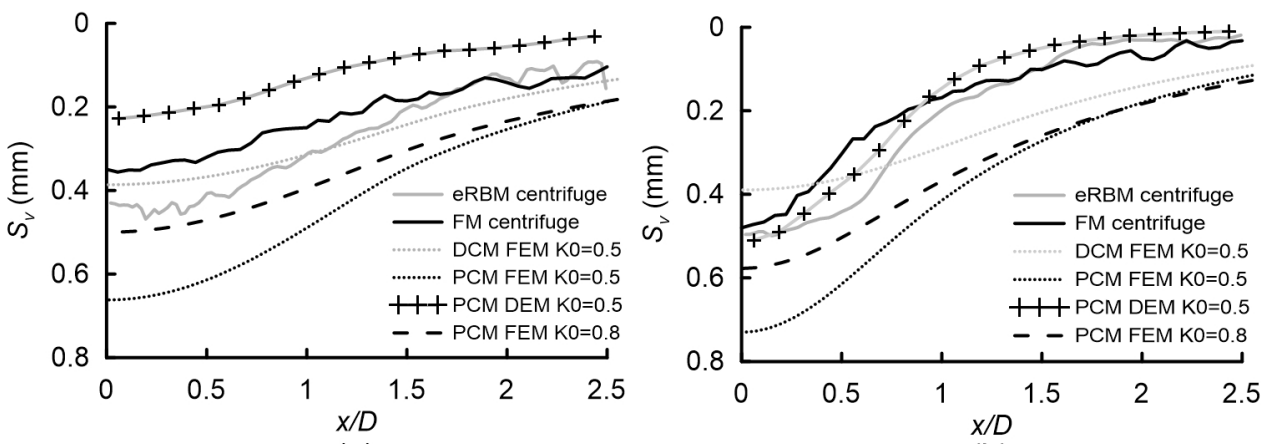

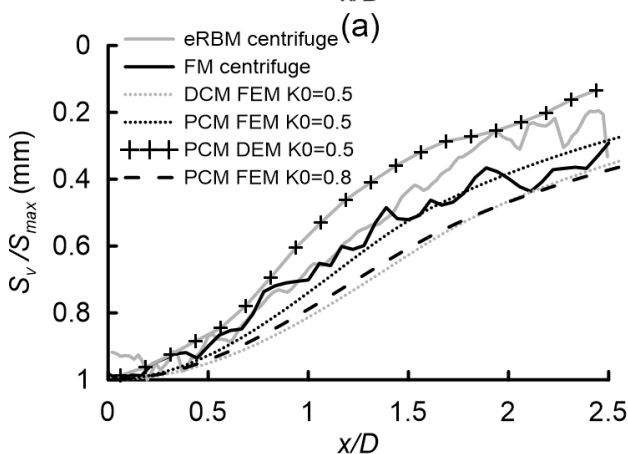

(c)

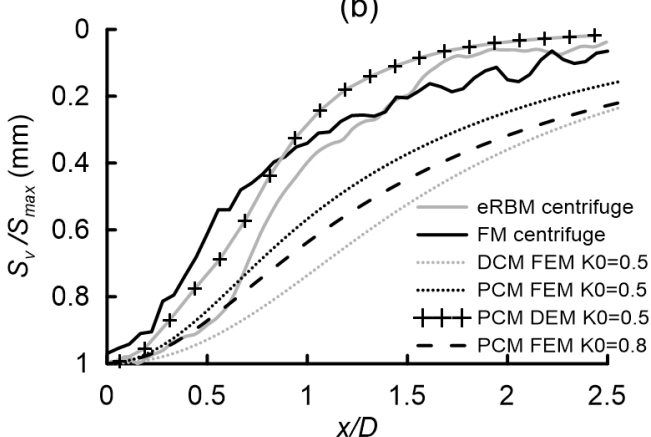

(d)

FiguRE 2. Vertical settlement trough for (a) surface (b) subsurface $\left(z / z_{t}=0.5\right)(\mathrm{c})$ normalised surface $(\mathrm{d})$ normalised subsurface $\left(z / z_{t}=0.5\right)$

\section{CONCLUSIONS}

The performance of continuum and discrete element numerical predictions of tunnelling induced greenfield ground movements was evaluated by comparing numerical analysis results against geotechnical centrifuge test data for dry sand. The type of boundary condition (pressure or displacement control) used in the tunnel to initiate ground displacements (volume loss) was shown to have a notable effect on ground movements in the centrifuge tests. Finite element method simulations were performed for both displacement and pressure controlled tunnels, and similarities were shown between the patterns of displacements between coinciding numerical and experimental data. The PCM FEM predictions over-predicted the magnitude of settlements when the commonly adopted assumption of $K_{0}=0.5$ was used; a better prediction of the magnitude of settlement was obtained for $K_{0}=0.5$. All the FEM predictions resulted in a wider settlement trough compared to the experimental data. The DEM analyses, which replicated a pressure control model 
tunnel, over-predicted the dilatancy of the experimental soil, however overall the predicted DEM settlement trough shape was better than the FEM models. These results indicate that DEM may prove a useful analysis tool for prediction of tunnelling induced ground displacements as well as soil-structure interaction problems related to tunnelling.

\section{References}

[1] I Boonsiri and J Takemura. Observation of ground movement with existing pile groups due to tunneling in sand using centrifuge modelling. Geotechnical and Geological Engineering, 33(3):621-640, 2015.

[2] G Song, A M Marshall, and C M Heron. A mechanical displacement control model tunnel for simulating eccentric ground loss in the centrifuge. In 9th International Conference of Physical Modelling in Geotechnics: ICPMG, 2018.

[3] C J Lee, B R Wu, H T Chen, and K H Chiang. Tunnel stability and arching effects during tunneling in soft clayey soil. Tunnelling and Underground Space Technology, 21(2):119-132, 2006.

[4] C Y Cheng, G R Dasari, Y K Chow, and C F Leung. Finite element analysis of tunnel-soil-pile interaction using displacement controlled model. Tunnelling and Underground Space Technology, 22(4):450-466, 2007.

[5] A M Marshall, I Elkayam, and A Klar. Ground behaviour above tunnels in sanddem simulations versus centrifuge test results. In Euro: Tun 2009, Proceedings of the 2nd International Conference on Computational Methods in Tunnelling, Bochum, Germany, pages 9-11. Aedificatio Verlag, Bochum, Germany, 2009.

[6] A M Marshall, R P Farrell, A Klar, and R Mair. Tunnels in sands: the effect of size, depth and volume loss on greenfield displacements. Géotechnique, 62(5):385-399, 2012 .

[7] A Franza. Tunnelling and its effects on piles and piled structures. PhD thesis, University of Nottingham, 2016.

[8] G Gudehus and D Mašín. Graphical representation of constitutive equations. Géotechnique, 59(2):147-151, 2009.

[9] G Lanzano, C Visone, E Bilotta, and F S de Magistris. Experimental assessment of the stress-strain behaviour of leighton buzzard sand for the calibration of a constitutive model. Geotechnical and Geological Engineering, 34(4):991-1012, 2016. 


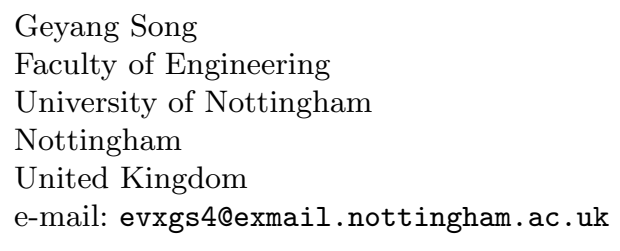

Andrea Franza

Department of Engineering

University of Cambridge

Cambridge

United Kingdom

e-mail: andreafranza@gmail.com

Itai Elkayam

Department of Structural Engineering

SCE- Shamoon College of Engioneering

e-mail: elkayam.itai@gmail.com

Alec M. Marshall

Faculty of Engineering

University of Nottingham

Nottingham

United Kingdom

e-mail: Alec.Marshall@nottingham.ac.uk

Assaf Klar

Faculty of Engineering

Technical University of Denmark

Lyngby

Denmark

e-mail: askla@byg.dtu.dk 